\title{
Coupled beam motion in a storage ring with crab cavities
}

\author{
Xiaobiao Huang* \\ SLAC National Accelerator Laboratory, Menlo Park, California 94025, USA
}

(Received 16 October 2015; published 16 February 2016)

\begin{abstract}
We studied the coupled beam motion in a storage ring between the transverse and longitudinal directions introduced by crab cavities. The analytic form of the linear decoupling transformation is derived. The equilibrium bunch distribution in an electron storage ring with a crab cavity is given, including contribution to the eigenemittance induced by the crab cavity. Application to the short pulse generation scheme using crab cavities is considered.
\end{abstract}

DOI: 10.1103/PhysRevAccelBeams.19.024001

\section{INTRODUCTION}

Crab cavities (also known as transverse deflecting cavities) have found major applications in storage rings. In colliders, they are used to rotate the colliding bunches at the collision point to create head-on collisions while the trajectories of the two beams cross at an angle (crab crossing) [1]. In electron storage rings, it has been proposed to use crab cavities to tilt a long bunch in the $y-z$ plane in order to select a short $\mathrm{x}$-ray pulse from the radiation generated by the beam with a vertical slit $[2,3]$.

The newly proposed two-frequency crab cavity scheme [3] differs from the original scheme [2] in several aspects. In particular, the crab cavity kicks on the short pulse generating bunches are not canceled within every revolution and hence these bunches are tilted everywhere in the ring in an equilibrium state. The equilibrium phase space distribution of these tilted bunches at the photon beam source points is critical for predicting the performance of the new short pulse scheme, which requires a thorough understanding of the beam dynamics with crab cavities.

A crab cavity gives the beam a time-dependent transverse kick. The kick is typically in the horizontal plane for the crab crossing application and in the vertical plane for the short pulse application. By virtue of the PanofskyWenzel theorem, the crab cavity also gives the beam a longitudinal kick that is dependent on the transverse offset. Naturally the crab cavity couples the transverse direction to the longitudinal direction. The nature of the linear coupled motion between the $y-z$ or $x-z$ directions is the same as the linear $x-y$ coupling introduced by a skew quadrupole. Therefore it can be likewise studied.

In some earlier work, the effects of the crab cavity on the beam are described as creating a $z$-dependent closed orbit

\footnotetext{
*xiahuang@slac.stanford.edu

Published by the American Physical Society under the terms of the Creative Commons Attribution 3.0 License. Further distribution of this work must maintain attribution to the author(s) and the published article's title, journal citation, and DOI.
}

$[3,4]$. This concept is valid when synchrotron motion is not included in the consideration. It can lead to useful results as synchrotron motion is usually slow for storage rings. However, a general analysis of particle motion with crab cavity should include synchrotron motion.

In this paper we study the coupled motion due to crab cavities in a storage ring through the transfer matrix. A matrix perturbation method is applied to find the linear transformation that block diagonalizes the one-turn transfer matrix. Analytic formulas for the decoupling linear transformation are derived. By applying the matrix perturbation technique to the Ohmi envelope equation [5] and considering the quantum diffusion of the beam with a tilted distribution on the $y-z$ directions, the equilibrium phase space distribution in an electron storage ring with crab cavities is also obtained. These results are applied to the short pulse generation scheme [3]. The short pulse performance is calculated and its functional dependence on crab cavity and lattice parameters is revealed. It is shown that the vertical eigenemittance due to the crab cavity induced tilt in bending magnets is a dominant factor that limits the achievable minimum pulse duration. Numeric example and simulation results for the short pulse generation application for SPEAR3 are presented.

In Sec. II we study the decoupling transformation for a vertical crab cavity. In Sec. III we first show the connection between the second order moment matrix of the original and decoupled coordinates. Then we derive the changes to the equilibrium distribution due to the crab cavity and calculate the short pulse performance for the short pulse generation scheme. A brief description of the procedure for decoupling the motion by a horizontal crab cavity is given in Sec. IV. Numeric examples and particle tracking are shown in Sec. V. The conclusion is given in Sec. VI.

\section{LINEAR COUPLING BY A VERTICAL CRAB CAVITY}

For a conventional crab cavity working at the TM110 mode, assuming the transverse deflection is on the vertical direction, the E-M fields are given by 


$$
E_{z}=\mathcal{E}_{0} k y \cos \omega t, \quad c B_{x}=\mathcal{E}_{0} \sin \omega t
$$

where $\mathcal{E}_{0} / c$ gives the amplitude of the magnetic induction, $k=\omega / c$ the angular wave number, $\omega=2 \pi f$ the angular frequency, and $c$ the speed of light. In crab cavity applications, the beam arrives at around $t=0$, where kick-to-time slope is the maximum. Correspondingly the kicks to the beam in linearized form are

$$
\Delta y^{\prime}=\frac{e V}{E} k z, \quad \Delta \delta=\frac{e V}{E} k y,
$$

where $\delta$ is the momentum deviation of the particle, $E$ is the beam energy and $V=\int_{\text {gap }} \mathcal{E}_{0} \sin (\omega t) c d t$ is the deflecting voltage. The linear motion through a crab cavity can be expressed via a transfer matrix of the coordinates $\mathbf{X}=\left(x, x^{\prime}, y, y^{\prime}, z, \delta\right)^{T}$. For a thin vertical crab cavity, the transfer matrix is given by

$$
\mathbf{T}_{c}=\left(\begin{array}{ccc}
\mathbf{I} & \mathbf{0} & \mathbf{0} \\
\mathbf{0} & \mathbf{I} & \epsilon \mathbf{W} \\
\mathbf{0} & \epsilon \mathbf{W} & \mathbf{I}
\end{array}\right)
$$

where $\mathbf{I}$ in this paper is the identity matrix of the appropriate size, $2 \times 2$ in this case, $\epsilon=\frac{e V k}{E}$, and

$$
\mathbf{W}=\left(\begin{array}{ll}
0 & 0 \\
1 & 0
\end{array}\right)
$$

When a crab cavity is inserted into the ring lattice, the one-turn transfer matrix will be changed. Assuming the crab cavity is located at point 2 , the one-turn transfer matrix at point 1 is

$$
\mathbf{T}_{1}=\mathbf{T}_{12} \mathbf{T}_{c} \mathbf{T}_{21}
$$

where $\mathbf{T}_{21}$ is the transfer matrix from point 1 to 2 and $\mathbf{T}_{12}$ the transfer matrix from point 2 to 1 . Using notations as defined in Ref. [6], the transfer matrix $\mathbf{T}_{21}$ can be written

$$
\mathbf{T}_{21}=\left(\begin{array}{ccc}
\mathbf{M}_{x, 21} & \mathbf{0} & \mathbf{E}_{21} \\
\mathbf{0} & \mathbf{M}_{y, 21} & \mathbf{0} \\
\mathbf{F}_{21} & \mathbf{0} & \mathbf{L}_{21}
\end{array}\right)
$$

where each element is a $2 \times 2$ matrix block. Transfer matrix $\mathbf{T}_{12}$ can be likewise expressed. Working out Eq. (5), we found

$$
\mathbf{T}_{1}=\mathbf{T}_{1}^{(0)}+\epsilon \tilde{\mathbf{T}}_{1},
$$

with
$\tilde{\mathbf{T}}_{1}=\left(\begin{array}{ccc}\mathbf{0} & \mathbf{E}_{12} \mathbf{W} \mathbf{M}_{y, 21} & \mathbf{0} \\ \mathbf{M}_{y, 12} \mathbf{W} \mathbf{F}_{21} & \mathbf{0} & \mathbf{M}_{y, 12} \mathbf{W L}_{21} \\ \mathbf{0} & \mathbf{L}_{12} \mathbf{W} \mathbf{M}_{y, 21} & \mathbf{0}\end{array}\right)$

where $\mathbf{T}_{1}^{(0)}$ is the one-turn matrix at point 1 without the crab cavity,

$$
\mathbf{T}_{1}^{(0)}=\mathbf{T}_{12} \mathbf{T}_{21}=\left(\begin{array}{ccc}
\mathbf{M}_{x} & \mathbf{0} & \mathbf{E} \\
\mathbf{0} & \mathbf{M}_{y} & \mathbf{0} \\
\mathbf{F} & \mathbf{0} & \mathbf{L}_{0}
\end{array}\right)
$$

In writing Eq. (9) we have neglected the synchrobetatron coupling effect that would be present if the rf cavity is located in a dispersive region [6]. This should not impact the results below as it usually only causes a small correction. The usual dispersion decoupling matrix is

$$
\mathbf{U}=\left(\begin{array}{ccc}
\mathbf{I} & \mathbf{0} & \mathbf{D}_{1} \\
\mathbf{0} & \mathbf{I} & \mathbf{0} \\
-\mathbf{D}_{1}^{+} & \mathbf{0} & \mathbf{I}
\end{array}\right)
$$

where $\mathbf{D}_{1}=\left(\mathbf{0}, \mathbf{d}_{1}\right), \mathbf{d}_{1}=\left(D_{1}, D_{1}^{\prime}\right)^{T}$, and the symplectic conjugate of matrix $\mathbf{D}_{1}$ is $\mathbf{D}_{1}^{+}=\mathbf{J}_{2}^{T} \mathbf{D}_{1}^{T} \mathbf{J}_{2}$, with

$$
\mathbf{J}_{2}=\left(\begin{array}{cc}
0 & 1 \\
-1 & 0
\end{array}\right) \text {. }
$$

Applying the transformation $\mathbf{U}$ to $\mathbf{T}_{1}$, we get a new transfer matrix for the betatron coordinates $\mathbf{X}=$ $\left(x_{\beta}, x_{\beta}^{\prime}, y, y^{\prime}, z, \delta\right)^{T}$,

$$
\begin{aligned}
\mathbf{T}_{1, n} & =\mathbf{U}^{-1} \mathbf{T}_{1} \mathbf{U} \\
& =\mathbf{T}_{1, n}^{(0)}+\epsilon \tilde{\mathbf{T}}_{1, n},
\end{aligned}
$$

where $x_{\beta}=x-D_{1} \delta, \quad x_{\beta}^{\prime}=x^{\prime}-D_{1}^{\prime} \delta, \quad \mathbf{T}_{1, n}^{(0)}$ is a blockdiagonal matrix,

$$
\mathbf{T}_{1, n}^{(0)}=\left(\begin{array}{ccc}
\mathbf{M}_{x} & \mathbf{0} & \mathbf{0} \\
\mathbf{0} & \mathbf{M}_{y} & \mathbf{0} \\
\mathbf{0} & \mathbf{0} & \mathbf{L}
\end{array}\right)
$$

and

$$
\tilde{\mathbf{T}}_{1, n}=\left(\begin{array}{ccc}
\mathbf{0} & \tilde{\mathbf{T}}_{x y} & \mathbf{0} \\
\tilde{\mathbf{T}}_{y x} & \mathbf{0} & \tilde{\mathbf{T}}_{y z} \\
\mathbf{0} & \tilde{\mathbf{T}}_{z y} & \mathbf{0}
\end{array}\right) .
$$

It has been shown that 


$$
\begin{aligned}
& \tilde{\mathbf{T}}_{x y}=-\mathbf{M}_{x, 12} \mathbf{D}_{2} \mathbf{W} \mathbf{M}_{y, 21}, \\
& \tilde{\mathbf{T}}_{y x}=\mathbf{M}_{y, 12} \mathbf{D}_{2}^{T}\left(\mathbf{M}_{x, 21}^{-1}\right)^{T} \mathbf{J}_{2}, \\
& \tilde{\mathbf{T}}_{y z}=\mathbf{M}_{y, 12} \mathbf{W} \mathbf{L}_{21, n}, \\
& \tilde{\mathbf{T}}_{z y}=\mathbf{L}_{12, n} \mathbf{W} \mathbf{M}_{y, 21},
\end{aligned}
$$

where $\mathbf{L}_{21, n}$ is longitudinal transfer matrix from point 1 to 2 with the $(1,2)$ element replaced with $\bar{\eta}_{21}$ as defined in Eq. (19) of Ref. [6] and likewise for $\mathbf{L}_{12, n}$.

From Eqs. (14)-(18) it is seen that the longitudinal motion and the vertical motion are coupled through the vertical crab cavity via the off-diagonal blocks $\tilde{\mathbf{T}}_{y z}$ and $\tilde{\mathbf{T}}_{z y}$. In addition, when the vertical crab cavity is located at a dispersive region [with nonzero horizontal dispersion $\left.\mathbf{D}_{2}=\left(D_{2}, D_{2}^{\prime}\right)^{T}\right]$, the horizontal and vertical motion are also coupled through the crab cavity.

The $x-y$ coupling and the $y-z$ coupling in Eq. (12) can be simultaneously diagonalized. The analytic form of the decoupling transformation can be derived with a matrix perturbation approach. Let the transformation be denoted by the matrix $\mathbf{V}$, i.e., $\mathbf{V}^{-1} \mathbf{T}_{1, n} \mathbf{V}$ is block diagonal. Because the matrix $\mathbf{T}_{1, n}$ deviates from the block diagonal matrix $\mathbf{T}_{1, n}^{(0)}$ by only a small amount that is proportional to $\epsilon$, we expect the deviation of $\mathbf{V}$ from the identity matrix to be proportional to $\epsilon$, too, i.e.,

$$
\mathbf{V}=\mathbf{I}+\tilde{\mathbf{V}},
$$

with $\tilde{\mathbf{V}} \propto \epsilon$. We use a trial form of $\tilde{\mathbf{V}}$,

$$
\tilde{\mathbf{V}}=\left(\begin{array}{ccc}
\mathbf{0} & \mathbf{C}_{1} & \mathbf{0} \\
-\mathbf{C}_{1}^{+} & \mathbf{0} & \mathbf{C}_{2} \\
\mathbf{0} & -\mathbf{C}_{2}^{+} & \mathbf{0}
\end{array}\right)
$$

It is easy to verify that the symplecticity of the matrix $\mathbf{V}$ is satisfied to first order of $\epsilon$ with $\tilde{\mathbf{V}}$ as given in Eq. (20). The transfer matrix after applying the $\mathbf{V}$ transformation is

$$
\begin{aligned}
\mathbf{V}^{-1} \mathbf{T}_{1, n} \mathbf{V} & \approx(\mathbf{I}-\tilde{\mathbf{V}})\left(\mathbf{T}_{1, n}^{(0)}+\epsilon \tilde{\mathbf{T}}_{1, n}\right)(\mathbf{I}+\tilde{\mathbf{V}}) \\
& =\mathbf{T}_{1, n}^{(0)}+\left(\epsilon \tilde{\mathbf{T}}_{1, n}-\tilde{\mathbf{V}} \mathbf{T}_{1, n}^{(0)}+\mathbf{T}_{1, n}^{(0)} \tilde{\mathbf{V}}\right)+O\left(\epsilon^{2}\right) .
\end{aligned}
$$

For the transfer matrix $\mathbf{V}^{-1} \mathbf{T}_{1, n} \mathbf{V}$ to be block diagonal to first order of $\epsilon$, we can require

$$
\epsilon \tilde{\mathbf{T}}_{1, n}-\tilde{\mathbf{V}} \mathbf{T}_{1, n}^{(0)}+\mathbf{T}_{1, n}^{(0)} \tilde{\mathbf{V}}=0
$$

because the diagonal blocks of the left-hand side of Eq. (22) are calculated to be all zeros. In fact, Eq. (22) is equivalent to

$$
\begin{array}{r}
\epsilon \tilde{T}_{x y}-\mathbf{C}_{1} \mathbf{M}_{y}+\mathbf{M}_{x} \mathbf{C}_{1}=0, \\
\epsilon \tilde{T}_{y x}+\mathbf{C}_{1}^{+} \mathbf{M}_{x}-\mathbf{M}_{y} \mathbf{C}_{1}^{+}=0, \\
\epsilon \tilde{T}_{y z}-\mathbf{C}_{2} \mathbf{L}+\mathbf{M}_{y} \mathbf{C}_{2}=0, \\
\epsilon \tilde{T}_{z y}+\mathbf{C}_{2}^{+} \mathbf{M}_{y}-\mathbf{L} \mathbf{C}_{2}^{+}=0 .
\end{array}
$$

From Eqs. (23) and (24) one can solve for $\mathbf{C}_{1}$, and similarly from Eqs. (25) and (26) for $\mathbf{C}_{2}$. The solutions are

$$
\begin{gathered}
\mathbf{C}_{1}=-\frac{\epsilon\left(\tilde{T}_{x y}+\tilde{T}_{y x}^{+}\right)}{\operatorname{Tr}\left(\mathbf{M}_{x}-\mathbf{M}_{y}\right)}, \\
\mathbf{C}_{2}=-\frac{\epsilon\left(\tilde{T}_{y z}+\tilde{T}_{z y}^{+}\right)}{\operatorname{Tr}\left(\mathbf{M}_{y}-\mathbf{L}\right)},
\end{gathered}
$$

where $\operatorname{Tr}(\cdot)$ denotes taking the trace of a matrix. The solution for $\mathbf{C}_{2}$ would be the same if we had block diagonalized the $y-z$ plane only, ignoring the $x-y$ coupling in Eq. (12). This indicates that the indirect $x-z$ coupling in Eq. (12) is a second order effect. It is worth noting that Eqs. (27) and (28) agree with the result of Ref. [7] to first order of $\epsilon$.

Inserting Eqs. (17) and (18) into Eq. (28), and expressing the related vertical and longitudinal transfer matrices in terms of the beta functions and phase advances, the four elements of

$$
\mathbf{C}_{2}=\left(\begin{array}{ll}
C_{11} & C_{12} \\
C_{21} & C_{22}
\end{array}\right)
$$

can be calculated and the results are

$$
\begin{aligned}
C_{11}= & \frac{\frac{1}{2} \epsilon \sqrt{\beta_{1} \beta_{2}}}{\cos 2 \pi \nu_{s}-\cos 2 \pi \nu_{y}}\left[\cos \Psi_{s, 12} \sin \left(2 \pi \nu_{y}-\Psi_{12}\right)\right. \\
& \left.+\cos \left(2 \pi \nu_{s}-\Psi_{s, 12}\right) \sin \Psi_{12}\right] \\
C_{12}= & \frac{\frac{1}{2} \epsilon \beta_{s} \sqrt{\beta_{1} \beta_{2}}}{\cos 2 \pi \nu_{s}-\cos 2 \pi \nu_{y}}\left[\sin \left(2 \pi \nu_{s}-\Psi_{s, 12}\right) \sin \Psi_{12}\right. \\
& \left.-\sin \Psi_{s, 12} \sin \left(2 \pi \nu_{y}-\Psi_{12}\right)\right] \\
C_{21}= & \frac{\frac{1}{2} \epsilon \sqrt{\beta_{2} / \beta_{1}}}{\cos 2 \pi \nu_{s}-\cos 2 \pi \nu_{y}}\left\{\cos \left(2 \pi \nu_{s}-\Psi_{s, 12}\right)\right. \\
& \times\left(\cos \Psi_{12}-\alpha_{1} \sin \Psi_{12}\right) \\
& \left.-\cos \Psi_{s, 12}\left[\cos \left(2 \pi \nu_{y}-\Psi_{12}\right)+\alpha_{1} \sin \left(2 \pi \nu_{y}-\Psi_{12}\right)\right]\right\}
\end{aligned}
$$




$$
\begin{aligned}
C_{22}= & \frac{\frac{1}{2} \epsilon \beta_{s} \sqrt{\beta_{2} / \beta_{1}}}{\cos 2 \pi \nu_{s}-\cos 2 \pi \nu_{y}}\left\{\sin \left(2 \pi \nu_{s}-\Psi_{s, 12}\right)\right. \\
& \times\left(\cos \Psi_{12}-\alpha_{1} \sin \Psi_{12}\right) \\
& \left.+\sin \Psi_{s, 12}\left[\cos \left(2 \pi \nu_{y}-\Psi_{12}\right)+\alpha_{1} \sin \left(2 \pi \nu_{y}-\Psi_{12}\right)\right]\right\},
\end{aligned}
$$

where $\Psi_{s, 12}$ is the synchrotron phase advance from point 2 to $1, \beta_{s}$ is the longitudinal beta function, $\nu_{s}$ is the synchrotron tune, $\nu_{y}$ is the vertical tune, $\alpha_{1}$ and $\beta_{1}$ are the vertical Courant-Snyder functions at point $1, \Psi_{12}$ is the vertical betatron phase advance from point 2 to 1 , and $\beta_{2}$ is the vertical beta function at point 2 . Because the longitudinal motion is slow, it can be ignored to simplify the results. The results under this assumption can be obtained from the exact formulas by using the approximations $\cos 2 \pi \nu_{s} \approx 1$ and $\beta_{s} \sin 2 \pi \nu_{s} \approx \bar{\eta}$, with $\bar{\eta}=-\oint D / \rho d s$. The simplified expressions for the $\mathbf{C}_{2}$ matrix elements are found to be

$C_{11}=\epsilon \frac{\sqrt{\beta_{1} \beta_{2}}}{2 \sin \pi \nu_{y}} \cos \left(\pi \nu_{y}-\Psi_{12}\right)$

$C_{12}=\epsilon \frac{\bar{\eta} \sqrt{\beta_{1} \beta_{2}}}{2 \sin \pi \nu_{y}}\left[\frac{\sin \Psi_{12}}{2 \sin \pi \nu_{y}}-\frac{\bar{\eta}_{12}}{\bar{\eta}} \cos \left(\pi \nu_{y}-\Psi_{12}\right)\right]$,

$C_{21}=\epsilon \frac{\sqrt{\beta_{2} / \beta_{1}}}{2 \sin \pi \nu_{y}}\left[\sin \left(\pi \nu_{y}-\Psi_{12}\right)-\alpha_{1} \cos \left(\pi \nu_{y}-\Psi_{12}\right)\right]$,

$$
\begin{aligned}
C_{22}= & \epsilon \frac{\bar{\eta} \sqrt{\beta_{2} / \beta_{1}}}{2 \sin \pi \nu_{y}}\left[\frac{1}{2 \sin \pi \nu_{y}}\left(\cos \Psi_{12}-\alpha_{1} \sin \Psi_{12}\right)\right. \\
& \left.-\frac{\bar{\eta}_{12}}{\bar{\eta}}\left[\sin \left(\pi \nu_{y}-\Psi_{12}\right)-\alpha_{1} \cos \left(\pi \nu_{y}-\Psi_{12}\right)\right]\right] .
\end{aligned}
$$

It is worth noting that

$$
\left\|\mathbf{C}_{2}\right\|=\frac{1}{8} \frac{\epsilon^{2} \beta_{2} \bar{\eta}}{\sin ^{2} \pi \nu_{y} \tan \pi \nu_{y}}
$$

which is a constant all around the ring.

In the above we showed that a vertical crab cavity causes $y-z$ coupling, and additionally $x-y$ coupling if it is located at a dispersive region. The coupled motioned can be decoupled with a linear transformation. The transformation for the $y-z$ coupling is given by Eqs. (19), (20), and (34)-(37).

\section{EQUILIBRIUM DISTRIBUTION \\ IN AN ELECTRON STORAGE RING WITH CRAB CAVITY}

\section{A. Beam distribution changes due to a crab cavity}

The decoupled coordinates $\mathbf{X}_{d}$ are related to the original coordinates $\mathbf{X}$ through $\mathbf{X}=\mathbf{U V X} \mathbf{X}_{d}$. The second order moment matrices of a particle distribution in $\mathbf{X}$ and $\mathbf{X}_{d}$ coordinates, defined as

$$
\Sigma=\left\langle\mathbf{X} \mathbf{X}^{T}\right\rangle, \quad \Sigma_{d}=\left\langle\mathbf{X}_{d} \mathbf{X}_{d}^{T}\right\rangle
$$

are related through

$$
\Sigma=\mathbf{U V} \Sigma_{\mathbf{d}} \mathbf{V}^{T} \mathbf{U}^{T}
$$

where $\langle\cdot\rangle$ denotes taking the average over the particle distribution and we have assumed the distribution is centered on the reference orbit. The moment matrices are symmetric. Matrix $\Sigma_{\mathbf{d}}$ is block diagonal. The two matrices $\Sigma$ and $\Sigma_{d}$ may be written as

$$
\Sigma=\left(\begin{array}{ccc}
\Sigma_{x x} & \Sigma_{x y} & \Sigma_{x z} \\
\Sigma_{x y}^{T} & \Sigma_{y y} & \Sigma_{y z} \\
\Sigma_{x z}^{T} & \Sigma_{y z}^{T} & \Sigma_{z z}
\end{array}\right)
$$

and

$$
\Sigma_{d}=\left(\begin{array}{ccc}
\Sigma_{x} & \mathbf{0} & \mathbf{0} \\
\mathbf{0} & \Sigma_{y} & \mathbf{0} \\
\mathbf{0} & \mathbf{0} & \Sigma_{z}
\end{array}\right) .
$$

Inserting Eqs. (10), (19), and (20) into Eq. (40), the block matrices in Eqs. (41) and (42) are related. It is found that the changes to the diagonal blocks of $\Sigma$ due to the crab cavity are second order effects, i.e., of the order $O\left(\epsilon^{2}\right)$, for example

$$
\Sigma_{y y}=\mathbf{C}_{1}^{+} \Sigma_{x}\left(\mathbf{C}_{1}^{+}\right)^{T}+\Sigma_{y}+\mathbf{C}_{2} \Sigma_{z} \mathbf{C}_{2}^{T}
$$

Therefore, it may be inferred that the deviation of the diagonal block matrices in $\Sigma_{d}$ from the case when the crab cavity is off (e.g., assuming it was adiabatically turned on) is also a second order effect. In other words, we assume

$$
\begin{aligned}
& \Sigma_{x} \approx \Sigma_{x 0}=\epsilon_{x}\left(\begin{array}{cc}
\beta_{x} & -\alpha_{x} \\
-\alpha_{x} & \gamma_{x}
\end{array}\right), \\
& \Sigma_{y} \approx \Sigma_{y 0}=\epsilon_{y}\left(\begin{array}{cc}
\beta_{y} & -\alpha_{y} \\
-\alpha_{y} & \gamma_{y}
\end{array}\right), \\
& \Sigma_{z} \approx \Sigma_{z 0}=\left(\begin{array}{cc}
\sigma_{z}^{2} & 0 \\
0 & \sigma_{\delta}^{2}
\end{array}\right),
\end{aligned}
$$


where $\epsilon_{x, y}$ are original horizontal and vertical emittances, $\alpha_{x, y}, \beta_{x, y}$, and $\gamma_{x, y}$ are the Courant-Snyder functions for the horizontal and vertical directions, with $\gamma_{x, y}=\left(1+\alpha_{x, y}^{2}\right) / \beta_{x, y}$, and $\sigma_{z}$ and $\sigma_{\delta}$ are original bunch length and momentum spread, respectively. This assumption is validated in the next subsection with the Ohmi envelope approach. In Eq. (46) we have assumed $\alpha_{s}=0$, i.e., there is no tilt between the $z-\delta$ directions. The deviation of matrix block $\Sigma_{x z}$ from the original case without crab cavity is also of the second order. Only $\Sigma_{x y}$ and $\Sigma_{y z}$ have first order dependence over the crab cavity strength parameter $\epsilon$,

$$
\begin{aligned}
\Sigma_{x y}= & -\Sigma_{x}\left(\mathbf{C}_{1}^{+}\right)^{T}+\left(\mathbf{C}_{1}-\mathbf{D}_{1} \mathbf{C}_{2}^{+}\right) \Sigma_{y}+\mathbf{D}_{1} \Sigma_{z} \mathbf{C}_{2}^{T}, \\
\Sigma_{y z}= & \mathbf{C}_{1}^{+} \Sigma_{x}\left(\mathbf{D}_{1}^{+}\right)^{T}-\Sigma_{y}\left[\left(\mathbf{C}_{2}^{+}\right)^{T}+\mathbf{C}_{1}^{T}\left(\mathbf{D}_{1}^{+}\right)^{T}\right] \\
& +\mathbf{C}_{2} \Sigma_{z} .
\end{aligned}
$$

We are interested in the tilt across the $y-z$ planes introduced by the crab cavity. In an electron storage ring, if originally there is no horizontal to vertical coupling, typically $\Sigma_{y 0} \approx 0$. Also, because the horizontal emittance is typically much smaller than the longitudinal emittance, unless the horizontal and vertical motions are near a resonance, normally the contribution from the $\Sigma_{x}$ term in Eq. (48) is much smaller than the last term. Keeping only the last term, we obtain

$$
\Sigma_{y z}=\left(\begin{array}{cc}
\sigma_{y z} & \sigma_{y \delta} \\
\sigma_{y^{\prime} z} & \sigma_{y^{\prime} \delta}
\end{array}\right) \approx \mathbf{C}_{2} \Sigma_{z}=\left(\begin{array}{ll}
C_{11} \sigma_{z}^{2} & C_{12} \sigma_{\delta}^{2} \\
C_{21} \sigma_{z}^{2} & C_{22} \sigma_{\delta}^{2}
\end{array}\right) .
$$

From Eq. (49) we see that the crab cavity causes a tilt of the beam distribution between the vertical and longitudinal directions. The tilt is not only between the vertical coordinates and the $z$-coordinate, but also the $\delta$-coordinate.

\section{B. First order perturbation to the equilibrium distribution by a crab cavity}

In an electron storage ring, the beam reaches an equilibrium distribution determined by the balance between quantum excitation and radiation damping. The equilibrium distribution at a location of the ring can be found by solving Ohmi's envelope equation [5],

$$
\mathbf{T}_{0} \Sigma_{0} \mathbf{T}_{0}^{T}+\overline{\mathbf{B}}_{0}=\Sigma_{0},
$$

where $\mathbf{T}_{0}$ is the one-turn transfer matrix (including damping), $\Sigma_{0}$ is the second order moment matrix as defined in Eq. (41), and $\overline{\mathbf{B}}_{0}$ is the one-turn integrated diffusion matrix

$$
\mathbf{B}_{0}\left(s_{0}\right)=\int_{s_{0}}^{s_{0}+C} T_{s_{0}+C, s^{\prime}} B\left(s^{\prime}\right) T_{s_{0}+C, s^{\prime}}^{T} d s^{\prime},
$$

where $T_{s_{0}+C, s^{\prime}}$ is the transfer matrix from $s^{\prime}$ to $s_{0}+C, C$ is the ring circumference, and $B\left(s^{\prime}\right)$ is the diffusion matrix at location $s=s^{\prime}$. In Eqs. (50) and (51) subscript 0 indicates the case without the crab cavity.

When the crab cavity is introduced to the ring, all quantities in Eq. (50) are changed. Suppose we are concerned of a point immediately downstream of the crab cavity, the envelope equation becomes

$$
\mathbf{T} \Sigma \mathbf{T}^{T}+\overline{\mathbf{B}}=\Sigma,
$$

with the new one-turn transfer matrix and new integrated diffusion matrix being

$$
\begin{aligned}
& \mathbf{T}=\mathbf{T}_{c} \mathbf{T}_{0}, \\
& \overline{\mathbf{B}}=\mathbf{T}_{c} \overline{\mathbf{B}}_{0} \mathbf{T}_{c}^{T} .
\end{aligned}
$$

Multiplying $\mathbf{T}_{c}^{-1}$ and $\left(\mathbf{T}_{c}^{T}\right)^{-1}$ from the left and right sides to Eq. (52), respectively, and inserting $\overline{\mathbf{B}}_{0}$ from Eq. (50), we get

$$
\mathbf{T}_{0}\left(\Sigma-\Sigma_{0}\right) \mathbf{T}_{0}^{T}=\mathbf{T}_{c}^{-1} \Sigma\left(\mathbf{T}_{c}^{T}\right)^{-1}-\Sigma_{0} .
$$

Rewriting $\mathbf{T}_{c}$ from Eq. (3) as

$$
\mathbf{T}_{c}=\mathbf{I}+\epsilon \tilde{\mathbf{W}}, \quad \tilde{\mathbf{W}}=\left(\begin{array}{ccc}
\mathbf{0} & \mathbf{0} & \mathbf{0} \\
\mathbf{0} & \mathbf{0} & \mathbf{W} \\
\mathbf{0} & \mathbf{W} & \mathbf{0}
\end{array}\right),
$$

the inverse matrices of $\mathbf{T}_{c}$ and its transpose are

$$
\begin{aligned}
\mathbf{T}_{c}^{-1} & =\mathbf{I}-\epsilon \tilde{\mathbf{W}}, \\
\left(\mathbf{T}_{c}^{T}\right)^{-1} & =\mathbf{I}-\epsilon \tilde{\mathbf{W}}^{T},
\end{aligned}
$$

with which Eq. (55) becomes

$$
\mathbf{T}_{0} \Delta \mathbf{T}_{0}^{T}-\boldsymbol{\Delta}=-\epsilon\left(\tilde{\mathbf{W}} \Sigma+\Sigma \tilde{\mathbf{W}}^{T}\right)+\epsilon^{2}\left(\tilde{\mathbf{W}} \Sigma \tilde{\mathbf{W}}^{T}\right),
$$

where we have used the definition

$$
\boldsymbol{\Delta}=\Sigma-\Sigma_{0} \cdot=\left(\begin{array}{ccc}
\boldsymbol{\Delta}_{x x} & \boldsymbol{\Delta}_{x y} & \boldsymbol{\Delta}_{x z} \\
\boldsymbol{\Delta}_{x y}^{T} & \boldsymbol{\Delta}_{y y} & \boldsymbol{\Delta}_{y z} \\
\boldsymbol{\Delta}_{x z}^{T} & \boldsymbol{\Delta}_{y z}^{T} & \boldsymbol{\Delta}_{z z}
\end{array}\right)
$$

Changes of the equilibrium distribution caused by the crab cavity can be found by solving Eq. (59) for $\boldsymbol{\Delta}$.

For results to first order of the strength parameter $\epsilon$, on the right-hand side (rhs) of Eq. (59) $\Sigma$ can be replaced by the original second order moment matrix, $\Sigma_{0}=\mathbf{U} \Sigma_{d 0} \mathbf{U}^{T}$, with $\Sigma_{d 0}$ a block diagonal matrix as in Eq. (42), and the $\epsilon^{2}$ term can be dropped. In this case, among the $2 \times 2$ subblocks of the rhs of Eq. (59), only the $x-y$ and $y-z$ blocks and their symmetric counterparts are nonzero. Therefore the elements in the sub-blocks $\boldsymbol{\Delta}_{x x}, \boldsymbol{\Delta}_{y y}, \boldsymbol{\Delta}_{z z}$, and $\boldsymbol{\Delta}_{x z}$ are 
solutions of a linear homogeneous equation set. In general this equation set is nondegenerate (the $\mathbf{T}_{0}$ matrix includes damping). Therefore these blocks are all zeros to the first order of $\epsilon$, which verifies the assumption we made in the previous subsection.

When the crab cavity is located in a dispersion region, the elements of $\boldsymbol{\Delta}_{x y}$ and $\boldsymbol{\Delta}_{y z}$ blocks are coupled in an inhomogeneous linear equation set,

$$
\begin{aligned}
& \mathbf{M}_{y} \Delta_{x y}^{T} \mathbf{M}_{x}^{T}+\mathbf{M}_{y} \boldsymbol{\Delta}_{y z} \mathbf{E}^{T}-\boldsymbol{\Delta}_{x y}^{T} \\
& \quad=(-\epsilon)\left(-\Sigma_{x 0}\left(\mathbf{D}_{1}^{+}\right)^{T} \mathbf{W}^{T}+\mathbf{D}_{1} \Sigma_{z 0} \mathbf{W}^{T}\right), \\
& \mathbf{M}_{x} \boldsymbol{\Delta}_{x y}^{T} \mathbf{F}^{T}+\mathbf{M}_{y} \boldsymbol{\Delta}_{y z} \mathbf{L}^{T}-\boldsymbol{\Delta}_{y z} \\
& \quad=(-\epsilon)\left(\mathbf{W} \Sigma_{z 0}+\mathbf{W} \mathbf{D}_{1}^{+} \Sigma_{x 0}\left(\mathbf{D}_{1}^{+}\right)^{T}+\Sigma_{y 0} \mathbf{W}^{T}\right),
\end{aligned}
$$

where $\Sigma_{x 0}, \Sigma_{y 0}$, and $\Sigma_{z 0}$ are diagonal blocks of $\Sigma_{d 0}$. For electron storage rings initially without $x-y$ coupling, the original vertical emittance is zero and hence $\Sigma_{y 0}=0$. Equations (61) and (62) can be solved for elements of $\boldsymbol{\Delta}_{x y}$ and $\boldsymbol{\Delta}_{y z}$. The coupling terms due to dispersion are on the order of $O\left(\mathcal{H}_{x} / \beta_{s}\right)$, which is usually very small, where $\mathcal{H}_{x}=\left[D^{2}+\left(\alpha_{x} D+\beta_{x} D^{\prime}\right)^{2}\right] / 2 \beta_{x}$ is the dispersion invariant. For example, SPEAR3 has $\beta_{s} \approx 6.2 \mathrm{~m}$ and at the standard straight sections $\mathcal{H}_{x}=1.0 \mathrm{~mm}$. Ignoring the coupling terms, the solution for elements of $\boldsymbol{\Delta}_{y z}$ is

$$
\begin{aligned}
\Sigma_{y z} & =\boldsymbol{\Delta}_{y z}=\mathbf{C}_{2} \Sigma_{z 0} \\
& \approx \frac{\epsilon \sigma_{z}^{2}}{2 \sin \pi \nu_{y}}\left(\begin{array}{cc}
\beta_{y} \cos \pi \nu_{y} & 0 \\
\sin \pi \nu_{y}-\alpha_{y} \cos \pi \nu_{y} & \frac{\bar{\eta} \gamma_{s}^{2}}{2 \sin \pi \nu_{y}}
\end{array}\right),
\end{aligned}
$$

where $\gamma_{s}=\sigma_{z} / \sigma_{\delta}$. This result is the same as given by Eqs. (34)-(37) and (49) for the location just downstream of the crab cavity.

\section{Vertical eigenemittance due to crab cavity}

When solving the Ohmi envelope equation, Eq. (52), for the equilibrium beam distribution with first order perturbation, we found that normal mode distributions do not change. However, because the longitudinal dimension of bunched beams in storage rings is usually much larger than the transverse dimensions, second order terms involving the longitudinal dimension may also be important. In fact, numeric solutions of Eq. (52) show that the normal mode distributions do have changes of order $\epsilon^{2}$. Notably, there is a finite vertical normal mode emittance (i.e., eigenemittance) and the bunch length changes.

Because coupling with the horizontal direction is small, we can consider only the vertical and longitudinal directions. Assuming the original vertical emittance is zero, $\Sigma_{y 0}=0$, and using

$$
\begin{aligned}
\boldsymbol{\Delta}_{z z} & =\left(\begin{array}{ll}
\Delta \sigma_{z z} & \Delta \sigma_{z \delta} \\
\Delta \sigma_{z \delta} & \Delta \sigma_{\delta \delta}
\end{array}\right), \quad \boldsymbol{\Delta}_{y z}=\left(\begin{array}{cc}
\sigma_{y z} & \sigma_{y \delta} \\
\sigma_{y^{\prime} z} & \sigma_{y^{\prime} \delta}
\end{array}\right), \\
\boldsymbol{\Delta}_{y y} & =\left(\begin{array}{cc}
\sigma_{y y} & \sigma_{y y^{\prime}} \\
\sigma_{y y^{\prime}} & \sigma_{y^{\prime} y^{\prime}}
\end{array}\right),
\end{aligned}
$$

the coupled matrix equations from Eq. (59) are given by

$$
\begin{aligned}
\mathbf{L} \boldsymbol{\Delta}_{z z} \mathbf{L}^{T}-\boldsymbol{\Delta}_{z z} & =(-\epsilon)\left(\begin{array}{cc}
0 & \sigma_{y z} \\
\sigma_{y z} & 2 \sigma_{y \delta}-\epsilon \sigma_{y y}
\end{array}\right) \\
\mathbf{M}_{y} \boldsymbol{\Delta}_{y z} \mathbf{L}^{T}-\boldsymbol{\Delta}_{y z} & =(-\epsilon)\left(\begin{array}{cc}
0 & \sigma_{y y} \\
\sigma_{z z} & \sigma_{z \delta}+\sigma_{y y^{\prime}}-\epsilon \sigma_{y z}
\end{array}\right) \\
\mathbf{M}_{y} \boldsymbol{\Delta}_{y y} \mathbf{M}_{y}^{T}-\boldsymbol{\Delta}_{y y} & =(-\epsilon)\left(\begin{array}{cc}
0 & \sigma_{y z} \\
\sigma_{y z} & 2 \sigma_{y^{\prime} z}-\epsilon \sigma_{z z}
\end{array}\right),
\end{aligned}
$$

where $\sigma_{z z}=\sigma_{z}^{2}=\sigma_{z 0}^{2}+\Delta \sigma_{z z}, \sigma_{z \delta}=\Delta \sigma_{z \delta}$, and $\sigma_{z 0}$ is the original bunch length.

In principle, solving Eqs. (65)-(67) for matrix elements in Eq. (64) gives the equilibrium distribution, from which one can calculate the eigenemittances. Although the general solution has not been found, approximate results can be derived from these equations. From Eqs. (65) and (66) we obtain

$$
\begin{aligned}
\Delta \sigma_{z z}-\beta_{s}^{2} \Delta \sigma_{\delta \delta} & =\sigma_{y z} \epsilon \beta_{s} \cot \psi_{s}, \\
\sigma_{y z} & \approx \sigma_{z z} \frac{\epsilon}{2} \frac{\beta_{y}}{\cos \psi_{s}-\cos \psi_{y}} .
\end{aligned}
$$

Numeric solutions indicate that $\beta_{s}^{2} \Delta \sigma_{\delta \delta} \ll \Delta \sigma_{z z}$. Ignoring the $\beta_{s}^{2} \Delta \sigma_{\delta \delta}$ term, we obtain

$\sigma_{z} \approx \sigma_{z 0}\left(1-\frac{\epsilon^{2}}{2} \beta_{s} \beta_{y} \cot \psi_{s} \frac{\sin \psi_{y}}{\cos \psi_{s}-\cos \psi_{y}}\right)^{-1 / 2}$.

The projected distribution on the $(z, \delta)$ plane, $\Sigma_{z z}$, and the longitudinal normal mode distribution, $\Sigma_{z}$, are approximately equal (their difference is a small term $\propto \epsilon^{2} \epsilon_{y}$, where $\epsilon_{y}$ is the vertical eigenemittance). Therefore, the longitudinal eigenemittance is

$$
\epsilon_{z} \approx \sigma_{z} \sigma_{\delta 0}
$$

where $\sigma_{z}$ is given in Eq. (70) and $\sigma_{\delta 0}$ is the original momentum spread.

A different approach has been taken to obtain the vertical eigenemittance due to the crab cavity. In an electron storage ring, the source of finite emittances is the stochastic photon emission in magnetic fields. The crab cavity causes a tilt across the longitudinal and vertical planes. Consequently, the energy loss of an electron due to photon emission will cause a random shift of vertical coordinates relative to its 
"ideal orbit," which gives rise to the vertical eigenemittance.

From Eq. (49) we find the average slope of the $y, y^{\prime}$ coordinates with respect to momentum deviation $\delta$,

$$
r_{y \delta}=\frac{\Delta y}{\Delta \delta}=C_{12}, \quad r_{y^{\prime} \delta}=\frac{\Delta y^{\prime}}{\Delta \delta}=C_{22} .
$$

The equivalent vertical coordinate displacements for an energy loss $\Delta \delta$ are thus

$$
\Delta y=C_{12} \Delta \delta, \quad \Delta y^{\prime}=C_{22} \Delta \delta,
$$

and the quadratic term of the Courant-Snyder invariant change is

$$
\Delta J_{y}=\mathcal{H}_{c} \Delta \delta^{2},
$$

where we defined crab cavity dispersion invariant,

$$
\mathcal{H}_{c}=\frac{1}{\beta_{y}}\left[C_{12}^{2}+\left(\alpha_{y} C_{12}+\beta_{y} C_{22}\right)^{2}\right] .
$$

The increase of vertical emittance due to photon emission in a tilted bunch is the same as due to vertical dispersion, except here the vertical dispersion invariant is replaced with the quantity $\mathcal{H}_{c}$.

Using Eqs. (31) and (33), and integrating $\mathcal{H}_{c}$ over the ring, we obtain the average crab cavity dispersion invariant,

$$
\left\langle\mathcal{H}_{c}\right\rangle=\frac{\epsilon^{2} \bar{\eta}^{2} \beta_{2}}{12} \frac{2+\cos 2 \pi \nu_{y}}{\left(\cos 2 \pi \nu_{s}-\cos 2 \pi \nu_{y}\right)^{2}},
$$

where we have assumed the synchrotron phase advance is linearly proportional to distance traveled in bending magnets. Similar to vertical emittance due to the usual vertical dispersion [8], the vertical eigenemittance for an isomagnetic storage ring due to crab cavity is given by

$$
\epsilon_{y}=C_{q} \frac{\gamma^{2}\left\langle\mathcal{H}_{c}\right\rangle}{J_{y} \rho}
$$

where $C_{q}=3.83 \times 10^{-13} \mathrm{~m}, \gamma$ is the Lorentz energy factor, $J_{y}=1$ is the vertical damping partition, and $\rho$ is bending radius.

\section{Prediction of short pulse performance}

For the crab cavity application of generating short pulses, an important task is to estimate the expected short pulse performance, such as the minimum pulse duration and the fraction of flux accepted by a slit with certain aperture. This can be done if we know the beam distribution at the source point and the photon beam optics between the source point and the slit.
The normal mode distributions for the longitudinal and vertical planes are Gaussian. Their projections onto the $y-z$ plane or $y^{\prime}-z$ plane are hence also Gaussian. Because the slit is usually placed far away from the source point, the tilt of the photon beam is primarily determined by the $y^{\prime}-z$ tilt at the source point. The distribution function for the $y^{\prime}-z$ projection can be written as

$\rho\left(y^{\prime}, z\right)=\frac{1}{2 \pi \epsilon_{y^{\prime} z}} \exp \left(-\frac{\sigma_{z}^{2} y^{\prime 2}-2 \sigma_{y^{\prime} z} y^{\prime} z+\sigma_{y^{\prime}}^{2} z^{2}}{2 \epsilon_{y^{\prime} z}^{2}}\right)$,

where $\epsilon_{y^{\prime} z}=\sqrt{\sigma_{z}^{2} \sigma_{y^{\prime}}^{2}-\sigma_{y^{\prime} z}}$ is the projected emittance. An ellipse that represents the $y^{\prime}-z$ distribution is shown in Fig. 1. The intercept with the $z$-axis signifies the minimum bunch length. It is given by

$$
\sigma_{z m}=\frac{\epsilon_{y^{\prime} z}}{\sigma_{y^{\prime}}}=\sigma_{z} \sqrt{1-\frac{\sigma_{y^{\prime} z}^{2}}{\sigma_{z}^{2} \sigma_{y^{\prime}}^{2}}} .
$$

With a finite vertical eigenemittance, the $y-z$ and $y-y$ blocks of the sigma matrix are related to the normal mode distribution through

$$
\begin{aligned}
& \Sigma_{y z}=\mathbf{C}_{2} \Sigma_{z}-\Sigma_{y}\left(\mathbf{C}_{2}^{+}\right)^{T}, \\
& \Sigma_{y y}=\mathbf{C}_{2} \Sigma_{z} \mathbf{C}_{2}^{T}+\Sigma_{y} .
\end{aligned}
$$

For simplicity we assume that at the source point $\alpha_{y}=0$, as this is usually the case. From Eqs. (80) and (81), we obtain

$$
\begin{aligned}
& \sigma_{y^{\prime} z}=C_{21} \sigma_{z}^{2}+C_{12} \frac{\epsilon_{y}}{\beta_{y}}, \\
& \sigma_{y^{\prime} y^{\prime}}=C_{21}^{2} \sigma_{z}^{2}+C_{22}^{2} \sigma_{\delta}^{2}+\frac{\epsilon_{y}}{\beta_{y}}+\sigma_{\theta}^{2},
\end{aligned}
$$

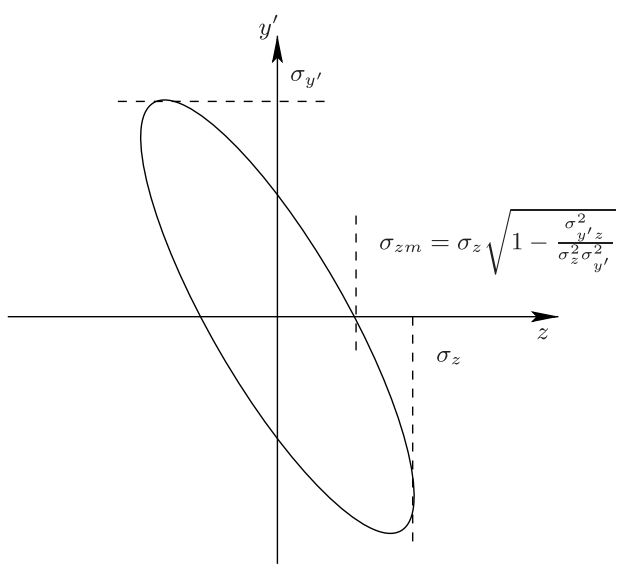

FIG. 1. The projected $y^{\prime}-z$ ellipse. 
where we have added the contribution of radiation divergence, $\sigma_{\theta}$, to $\sigma_{y^{\prime} y^{\prime}}$, such that Eqs. (82) and (83) are for the photon beam distribution at the source point.

Ignoring small terms, the projected emittance on the $y^{\prime}-z$ plane is given by

$$
\epsilon_{y^{\prime} z}=\sigma_{z}\left(C_{22}^{2} \sigma_{\delta}^{2}+\frac{\epsilon_{y}}{\beta_{y}}+\sigma_{\theta}^{2}\right)^{1 / 2} .
$$

The minimum pulse duration is thus

$$
\begin{aligned}
\sigma_{z m} & =\sigma_{z}\left(\frac{C_{22}^{2} \sigma_{\delta}^{2}+\frac{\epsilon_{y}}{\beta_{y}}+\sigma_{\theta}^{2}}{C_{21}^{2} \sigma_{z}^{2}+C_{22}^{2} \sigma_{\delta}^{2}+\frac{\epsilon_{y}}{\beta_{y}}+\sigma_{\theta}^{2}}\right)^{1 / 2} \\
& \approx \frac{1}{C_{21}} \sqrt{\frac{\epsilon_{y}}{\beta_{y}}+\sigma_{\theta}^{2}}
\end{aligned}
$$

where the approximate equality is equivalent to Eq. (8) of Ref. [3] and is valid when the $\sigma_{\delta}^{2}$ term is negligible. For hard $\mathrm{x}$-ray sources, the radiation divergence term may also be negligible. In this case, if there are no other sources of vertical emittance, the minimum pulse duration is independent of the strength of the crab cavity. With a low deflecting voltage, the $y-z$ tilt of the photon beam at the slit is small, which requires a small slit aperture for a given accepted flux. Of course, in reality a reasonable deflecting voltage is needed to overcome the contribution of the finite vertical emittance due to spurious vertical dispersion and horizontal to vertical coupling and the finite radiation divergence. An optimal deflecting voltage is probably achieved when the term of the crab cavity induced eigenemittance is a few times of the contributions of original vertical emittance and radiation divergence. The longitudinal distribution of the short pulse accepted by a given slit aperture is calculated from the distribution function $\rho\left(y^{\prime}, z\right)$ with

$$
\lambda\left(z ; y_{a}\right)=\int_{-y_{a} / L_{a}}^{y_{a} / L_{a}} d y^{\prime} \rho\left(y^{\prime}, z\right)
$$

where $y_{a}$ is the half aperture, $L_{a}$ is the distance from the slit to the source point, and we have ignored the finite vertical size of the electron beam at the source point. The percentage of total flux in the accepted pulse can be calculated with

$$
F\left(y_{a}\right)=\int d z \lambda\left(z ; y_{a}\right)
$$

and the pulse duration $\sigma_{z p}$ can be obtained from

$$
\sigma_{z p}^{2}\left(y_{a}\right)=\frac{\int d z z^{2} \lambda\left(z ; y_{a}\right)}{\int d z \lambda\left(z ; y_{a}\right)}
$$

\section{COUPLED MOTION BY A HORIZONTAL CRAB CAVITY}

Because of the lack of vertical dispersion, the coupling due to a horizontal crab cavity generally does not involve the vertical plane. Therefore we only need to study the 4D phase space coordinates, $\left(x, x^{\prime}, z, \delta\right)$. The transfer matrix for the crab cavity is

$$
\mathbf{T}_{c}=\mathbf{I}+\epsilon \mathbf{W}_{x}, \quad \tilde{\mathbf{W}}_{x}=\left(\begin{array}{cc}
\mathbf{0} & \mathbf{W} \\
\mathbf{W} & \mathbf{0}
\end{array}\right) .
$$

Assuming the crab cavity is located at point 2, the one-turn transfer matrix at point 1 is

$$
\mathbf{T}_{1}=\mathbf{T}_{12} \mathbf{T}_{c} \mathbf{T}_{21}=\mathbf{T}_{1}^{(0)}+\epsilon \tilde{\mathbf{T}}_{1},
$$

with

$\tilde{\mathbf{T}}_{1}=\left(\begin{array}{cc}\mathbf{E}_{12} \mathbf{W} \mathbf{M}_{21}+\mathbf{M}_{12} \mathbf{W} \mathbf{F}_{21} & \mathbf{E}_{12} \mathbf{W} \mathbf{E}_{21}+\mathbf{M}_{12} \mathbf{W} \mathbf{L}_{21} \\ \mathbf{L}_{12} \mathbf{W} \mathbf{M}_{21}+\mathbf{F}_{12} \mathbf{W} \mathbf{F}_{21} & \mathbf{L}_{12} \mathbf{W} \mathbf{E}_{21}+\mathbf{F}_{12} \mathbf{W F}_{21}\end{array}\right)$,

where we dropped subscript $x$ for $\mathbf{M}_{12}$ and $\mathbf{M}_{21}$. Then the dispersion decoupling transformation (the $\mathbf{U}$ matrix) can be applied, followed by a second decoupling transformation V. The same procedure can be carried out as for the vertical crab cavity case. It is noted that if the crab cavity location (point 2) is dispersion free, then the decoupling transfer matrix $\mathbf{V}$ is the same as the $y-z$ plane for the vertical case. Equations (28)-(37) are valid with the $y$-plane parameters replaced by the horizontal counterparts.

\section{NUMERIC EXAMPLE AND SIMULATION}

We use the SPEAR3 storage ring lattice to work out a numeric example in order to illustrate the results derived in the previous sections. Table I lists a few related parameters of the machine. The method of using two crab cavities with different frequencies to tilt the beam in the $y-z$ plane for the generation of short $\mathrm{x}$-ray pulse is considered [3]. For example, if the frequencies of the two crab cavities are 6 and 6.5 times of the rf frequency of the ring, respectively,

TABLE I. Selected parameters of SPEAR3.

\begin{tabular}{lcc}
\hline \hline Parameters & Value & Unit \\
\hline Energy & 3 & $\mathrm{GeV}$ \\
Circumference & 234.1 & $\mathrm{~m}$ \\
Tune $\nu_{x, y}$ & $14.106,6.177$ & \\
rf frequency $f_{\mathrm{rf}}$ & 476.3 & $\mathrm{MHz}$ \\
Bunch length $\sigma_{z}$ & 6.0 & $\mathrm{~mm}$ \\
Momentum spread $\sigma_{\delta}$ & 0.001 & \\
Momentum compaction $\alpha_{c}$ & $1.62 \times 10^{-3}$ & \\
Synchrotron tune $\nu_{s}$ & 0.010 & \\
\hline \hline
\end{tabular}


and the deflecting voltages are properly matched, the tilting effects of the two crab cavities cancel for half of the buckets. For the other half buckets, the tilting effects add up. For a bunch in a tilted bucket, the linear dynamics is not different from the case with one crab cavity-only that the strength parameter now is the sum of the two crab cavities, $\epsilon=e\left(V_{1} k_{1}+V_{2} k_{2}\right) / E_{0}$, where $V_{1,2}$ and $k_{1,2}$ are the deflecting voltage and angular wave number for the two crab cavities, respectively.

In the following we consider only one crab cavity, with a deflecting voltage of $V=2 \mathrm{MV}$ and the frequency is $f_{1}=6 f_{\text {rf }}=2857.8 \mathrm{MHz}$. The strength parameter is thus $\epsilon=-0.0399$, where there is a negative sign due to the choice of crab cavity phase. The crab cavity is located in one of the matching straight sections, where $\beta_{y}=2.803 \mathrm{~m}$, $\alpha_{y}=-0.348, D_{x}=0.085$, and $D_{x}^{\prime}=-0.001$.

We consider an observation point at the center of a standard straight section (13S), where $\beta_{y}=4.860 \mathrm{~m}$, $\alpha_{y}=0.0, D_{x}=0.10, D_{x}^{\prime}=0.0$, and the vertical betatron phase advance from the crab cavity to $13 \mathrm{~S}$ is $\Delta \Psi_{y}=$ $5.3761 \mathrm{rad}$ modulo $2 \pi$. The one-turn transfer matrix at this point is

$$
\begin{aligned}
& \mathbf{T}_{13 S} \\
& =\left(\begin{array}{cccccc}
0.7860 & 5.4926 & 0.002 & -0.0213 & 0.001 & 0.019 \\
-0.0699 & 0.7838 & 0.000 & -0.0007 & 0.000 & 0.007 \\
0.0010 & -0.0137 & 0.439 & 4.3579 & 0.116 & -0.014 \\
-0.0002 & 0.0022 & -0.189 & 0.4430 & -0.019 & 0.002 \\
-0.0067 & -0.0224 & -0.003 & 0.0346 & 0.997 & -0.379 \\
0.0 & -0.0008 & 0.013 & -0.1329 & 0.010 & 0.999
\end{array}\right) .
\end{aligned}
$$

Following the equations in Sec. II, the matrices $\mathbf{C}_{1}$ and $\mathbf{C}_{2}$ are calculated as

$$
\begin{aligned}
& \mathbf{C}_{1}=\epsilon\left(\begin{array}{cc}
-0.0452 & -0.2162 \\
0.0083 & 0.0109
\end{array}\right), \\
& \mathbf{C}_{2}=\epsilon\left(\begin{array}{ll}
0.3799 & 1.0904 \\
0.7146 & 0.0268
\end{array}\right),
\end{aligned}
$$

and the new transfer matrix is

$$
\begin{aligned}
& \mathbf{V}^{-1} \mathbf{U}^{-1} \mathbf{T}_{13 \mathrm{~S}} \mathbf{U} \mathbf{V} \\
& =\left(\begin{array}{cccccc}
0.7860 & 5.4926 & 0.0 & 0.0 & 0.0001 & 0.0 \\
-0.0699 & 0.7838 & 0.0 & 0.0 & 0.0 & 0.0 \\
0.0 & 0.0 & 0.440 & 4.3526 & 0.0002 & 0.0 \\
0.0 & 0.0 & -0.185 & 0.4439 & -0.0002 & 0.0 \\
0.0 & 0.0 & 0.0 & 0.0002 & 0.9963 & -0.379 \\
0.0001 & 0.0 & -0.0 & -0.0008 & 0.0138 & 0.998
\end{array}\right) .
\end{aligned}
$$

The off-diagonal blocks of the new transfer matrix are substantially reduced toward zero, which verifies the results in Sec. II.

We also performed a particle tracking simulation to determine the equilibrium distribution with the crab cavity in the lattice. There is no $x-y$ coupling in the model originally without the crab cavity. Sextupoles in the lattice are set to correct both the horizontal and vertical chromaticities to 2. Simulation is done with the tracking code ACCELERATOR TOOLBOX [9], with new functions added to model the crab cavity and quantum excitation. The code ELEGANT [10] is also used for tracking and good agreement is found between the two codes. All particles are launched with zero coordinate offsets and tracked for 30000 turns, which are 7.5 times of the longitudinal damping time and 4.4 times of the vertical damping time.

The projection of the phase space volume onto the $y-z$ and $y^{\prime}-z$ planes are shown in Fig. 2 for the $13 \mathrm{~S}$ observation point. Also plotted in the figure are ellipses calculated with Ohmi envelope ("Ohmi") and with the corresponding second order moments calculated with Eqs. (80) and (81) and Eqs. (70) and (77) ("Formulas"). The area of the ellipses are 6 times of the respective projected emittances. Ellipses derived with Ohmi envelope calculation overlap almost exactly with the ones calculated with
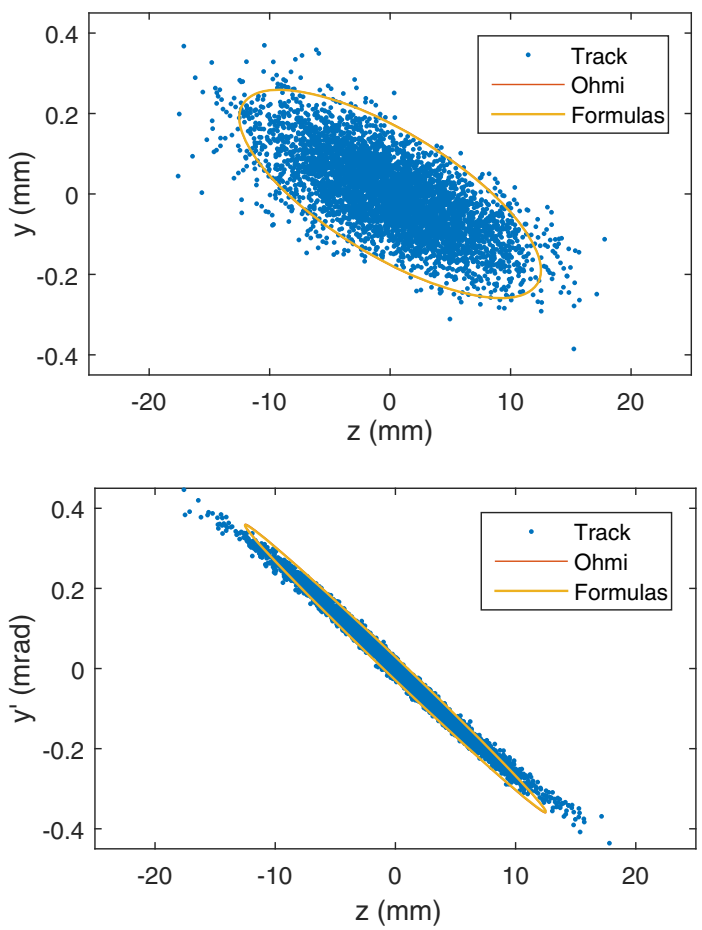

FIG. 2. The projection of equilibrium particle distribution (blue dots) at $13 \mathrm{~S}$ onto the $y-z$ plane (top) and $y^{\prime}-z$ plane (bottom) are compared to ellipses from numeric Ohmi envelope calculation (Ohmi) and calculations with Eqs. (80) and (81) and Eqs. (70) and (77) (Formulas). The ellipses cover $6 \sigma$ of the Gaussian distribution for the corresponding projected emittances. 
TABLE II. Tilts and projected emittances at $13 \mathrm{~S}$ for the numeric example as shown in Fig. 2.

\begin{tabular}{lrrrc}
\hline \hline Parameters & Formulas & \multicolumn{1}{c}{ Ohmi } & Track & Unit \\
\hline$\Delta y / \Delta z$ & -0.0152 & -0.0151 & -0.0136 & \\
$\Delta y^{\prime} / \Delta z$ & -0.0285 & -0.0287 & -0.0270 & $\mathrm{rad} / \mathrm{m}$ \\
$\epsilon_{y z}$ & 0.3668 & 0.3696 & 0.3620 & $\mathrm{~mm}^{2}$ \\
$\epsilon_{y^{\prime} z}$ & 0.0610 & 0.0615 & 0.0709 & $\mathrm{~mm}-\mathrm{mrad}$ \\
\hline \hline
\end{tabular}

formulas. Table II compares the tilts and projected emittances in $y-z$ and $y^{\prime}-z$ planes, which serves as a numeric characterization of the tracking data and calculations shown in Fig. 2.

Figure 2 and Table II show excellent agreement between numeric Ohmi envelope calculation and the analytic calculation. Both calculations generally agree with particle tracking data while there are some small differences. It is worth noting that the analytic results are based on a linear theory of the crab cavity and storage ring optics. The linear theory approaches the numeric Ohmi envelope calculation at its best, a goal that appears to be nearly achieved. The differences between the calculations and particle tracking may come from the nonlinearity of the sinusoidal wave of the crab cavity and the nonlinearity in the storage ring optics, which are to be investigated.

To check the formulas for bunch length [Eq. (70)] and vertical eigenemittance [Eq. (77)], we did numeric
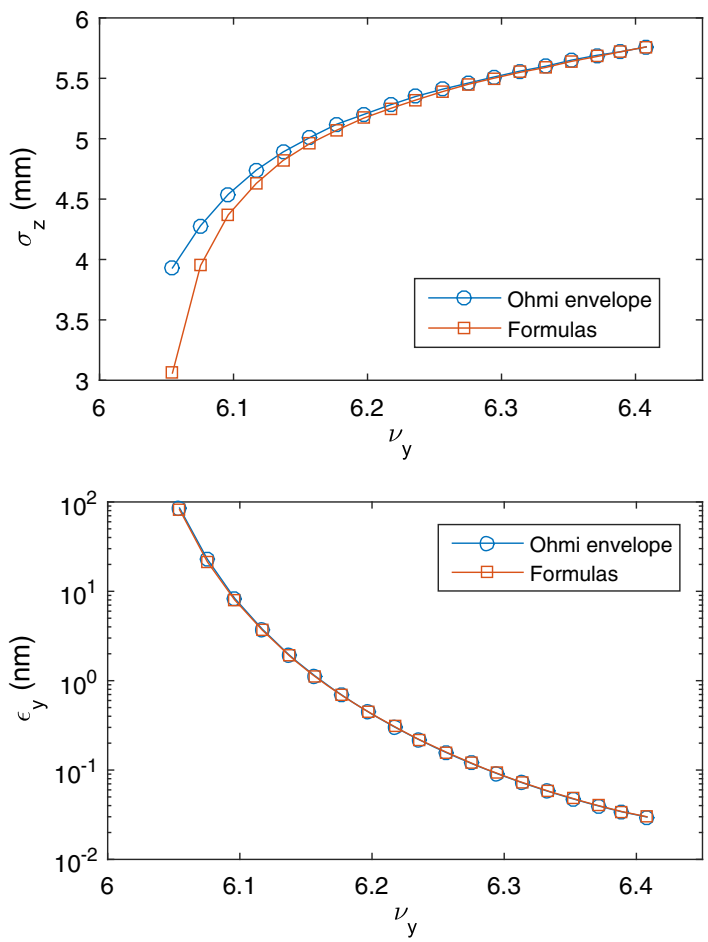

FIG. 3. Bunch length (top) and vertical eigenemittance (bottom) from numeric Ohmi envelope calculation is compared to formulas [Eq. (70) for bunch length and Eq. (77) for vertical eigenemittance].

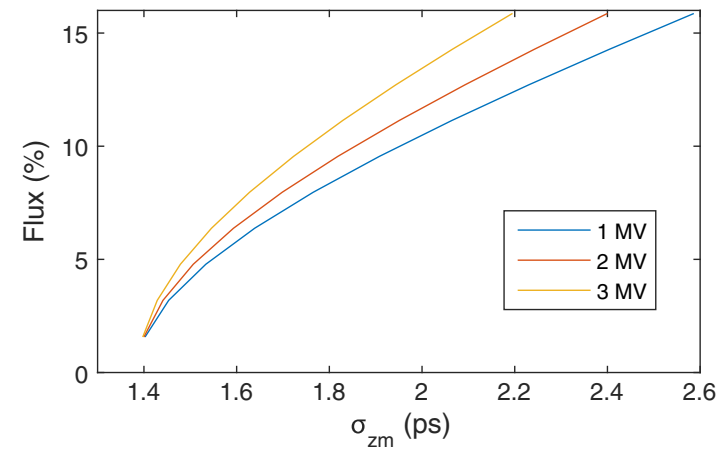

FIG. 4. Percentage of flux vs pulse duration for various deflecting voltages.

calculation of the Ohmi envelope while varying the vertical tune of the lattice. The results are compared to calculations by the formulas and are shown in Fig. 3. It is seen that the semiempirical formula, Eq. (70), agrees with numeric calculations for large tune separation between the vertical and longitudinal directions, but deviates from numeric results as the vertical tune approaches the synchrotron tune. However, the analytic formula, Eq. (77), agrees with numeric results excellently in the entire parameter range.

The minimum pulse duration is typically dominated by the vertical eigenemittance term in Eq. (85). The percentage of flux for short pulses accepted by a slit as a function of pulse duration is plotted in Fig. 4 for various deflecting voltages. No original vertical emittance or radiation divergence is assumed. In this case, indeed a higher deflecting voltage does not reduce the minimum pulse duration, although it helps reduce the pulse duration for a given percentage of flux.

Because the vertical eigenemittance due to the crab cavity is strongly dependent on the momentum compaction factor [see Eqs. (76) and (77)], the minimum pulse duration is expected to be sensitive to momentum compaction factor changes. Figure 5 compares the minimum pulse duration vs vertical tune for two SPEAR3 lattices with crab cavity parameters, crab cavity and source point locations as given in the above example. Parameters for the "low emittance"

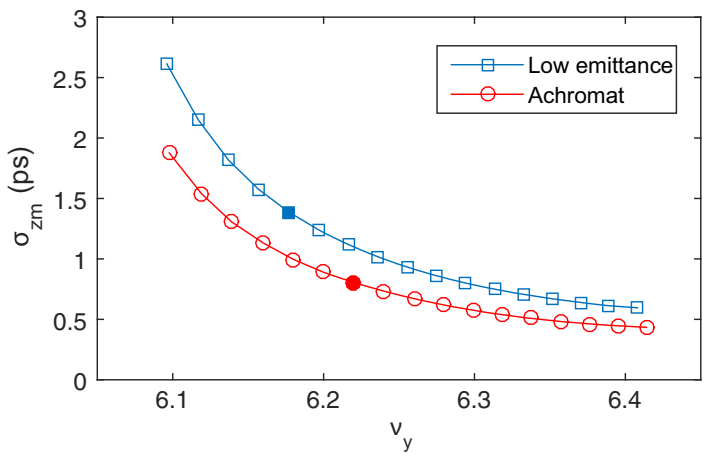

FIG. 5. Minimum pulse duration vs vertical tune for the low emittance lattice and the achromat lattice of SPEAR3. Filled markers indicate nominal tunes for the lattices. 
lattice are listed in Table I. The "achromat" lattice has a nominal vertical tune $\nu_{y}=6.22$, a momentum compaction factor $\alpha_{c}=1.18 \times 10^{-3}$, and a nominal bunch length of $\sigma_{z}=5.0 \mathrm{~mm}$. Clearly lowering the momentum compaction factor helps reduce the pulse duration, by a factor more than the reduction of nominal bunch length. Increasing the vertical tune reduces the minimum pulse duration, although the return diminishes as the tune shifts up.

\section{CONCLUSION}

We studied the linear coupling between the transverse and longitudinal directions introduced by a crab cavity in a storage ring. A matrix perturbation method is applied to derive the transformation that decouples the $6 \mathrm{D}$ one-turn transfer matrix. Analytic formulas are given for the coefficients of the decoupling transformation. The equilibrium particle distribution in an electron storage ring is also derived by applying the perturbation method to the Ohmi envelope equation [5]. Considering the quantum excitation in bending magnets for a beam distribution with tilt across the $y-z$ directions, we derived the vertical eigenemittance due to crab cavities. Application to the short pulse generation scheme using crab cavities is considered. Numeric example and particle tracking are shown to demonstrate the analytic results.

\section{ACKNOWLEDGMENTS}

Discussions with Sasha Zholents and James Safranek were helpful. The study is supported by DOE Contract No. DE-AC02-76SF00515.

[1] K. Oide and K. Yokoya, Phys. Rev. A 40, 315 (1989).

[2] A. Zholents, P. Heimann, M. Zolotorev, and J. Byrd, Nucl. Instrum. Methods Phys. Res., Sect. A 425, 385 (1999).

[3] A. Zholents, Nucl. Instrum. Methods Phys. Res., Sect. A 798, 111 (2015).

[4] Y.-P. Sun, R. Assmann, J. Barranco, R. Tomás, T. Weiler, F. Zimmermann, R. Calaga, and A. Morita, Phys. Rev. ST Accel. Beams 12, 101002 (2009).

[5] K. Ohmi, K. Hirata, and K. Oide, Phys. Rev. E 49, 751 (1994).

[6] X. Huang, Phys. Rev. ST Accel. Beams 10, 014002 (2007).

[7] D. Sagan and D. Rubin, Phys. Rev. ST Accel. Beams 2, 074001 (1999).

[8] S. Y. Lee, Accelerator Physics (World Scientific, Singapore, 1999).

[9] A. Terebilo, Report No. SLAC-PUB-8732, 2001.

[10] M. Borland, APS Report No. LS-287. 